\title{
SMURF1-mediated ubiquitination of ARHGAP26 promotes ovarian cancer cell invasion and migration
}

\author{
Xuri Chen', Shaoyun Chen², Yao Li', Yanling Gao', Shuying Huang ${ }^{1}$, Hongping Li ${ }^{3}$ and Yuanfang Zhu ${ }^{1,2}$
}

\begin{abstract}
Rho GTPase-activating protein 26 (ARHGAP26) is a negative regulator of the Rho family that converts the small GTPbinding protein RhoA (GTP-RhoA) to its inactive GDP-bound form and is a putative tumor suppressor gene associated with cell growth and migration. Here, the involvement of ARHGAP26 in ovarian cancer cell proliferation and migration was investigated. In this study, low ARHGAP26 expression was observed in ovarian cancer tissues and was associated with a poor overall survival and higher $\beta$-catenin expression in patients with ovarian cancer. A2780 and HEY cells with ARHGAP26 upregulation showed decreased cell proliferation, migration, and invasion, along with decreased GTPRhoA, $\beta$-catenin, VEGF, MMP2, and MMP7 expression. ARHGAP26 upregulation in A2780 cells also inhibited lung metastasis in vivo. SKOV3 cells with ARHGAP26 downregulation demonstrated an inverse effect, which was inhibited by ARHGAP26 overexpression or DKK1, an antagonist of the $\beta$-catenin pathway. SMURF1, an E3 ubiquitin ligase, interacted with and induced ubiquitination of ARHGAP26. ARHGAP26 upregulation in SKOV3 cells significantly inhibited SMURF1 upregulation-induced cell migration and invasion. Overall, SMURF1-mediated ubiquitination of ARHGAP26 may promote invasion and migration of ovarian cancer cells via the $\beta$-catenin pathway.
\end{abstract}

\section{Introduction}

Ovarian cancer is one of the three most common malignant tumors of female reproductive organs and the predominant pathological type is epithelial carcinoma, accounting for $85-90 \%$ of malignant ovarian tumors ${ }^{1}$. After cervical and endometrial cancer, the incidence of ovarian cancer ranks third, but the mortality rate is the highest compared with other malignant tumors of female reproductive organs ${ }^{2}$. More than 200,000 new cases are diagnosed and 100,000 people die each year ${ }^{3}$. The 5 -year overall survival rate of ovarian cancer patients is only $47 \%$ due to the lack of early clinical symptoms, practical early

Correspondence: Hongping Li (hongping_li@126.com) or Yuanfang Zhu (zhuzn0620@163.com)

${ }^{1}$ Department of Obstetrics and Gynecology, Bao'an Maternity and Child Health Hospital, Jinan University, Shenzhen 518100, China

${ }^{2}$ Maternal-Fetal Medicine Institute, Bao'an Maternity and Child Health Hospital, Jinan University, Shenzhen 518100, China

Full list of author information is available at the end of the article.

These authors contributed equally: Xuri Chen, Shaoyun Chen diagnosis, and lasting and effective treatment, as well as the high susceptibility to chemotherapy resistance and relapse $^{4,5}$. Therefore, a better understanding of the potential pathogenic genes is urgently required for improved ovarian cancer treatment.

Rho GTPase-activating protein 26 (ARHGAP26) belongs to the small $\mathrm{G}$ protein family that can hydrolyze active Rho GTPase into inactive Rho GDP and negatively regulates RhoA ${ }^{6}$. RhoA belongs to the Rho GTPase superfamily and is abnormally expressed in many malignant tumors and involved in the tumor invasion and metastasis ${ }^{7,8}$, contributing to the occurrence and development of cancer. ARHGAP26 is a recognized tumor suppressor gene that was found inactivated in acute myeloid leukemia and an independent prognostic factor for acute myeloid leukemia ${ }^{9}$. Deletion and mutation of ARHGAP26 can lead to promyelocytic leukemia ${ }^{10}$, suggesting tumor suppressive activity of ARHGAP26. ARHGAP26 was downregulated in glioblastoma and associated

\section{(c) The Author(s) 2019}

(c) (i) Open Access This article is licensed under a Creative Commons Attribution 4.0 International License, which permits use, sharing, adaptation, distribution and reproduction cc) in any medium or format, as long as you give appropriate credit to the original author(s) and the source, provide a link to the Creative Commons license, and indicate if changes were made. The images or other third party material in this article are included in the article's Creative Commons license, unless indicated otherwise in a credit line to the material. If material is not included in the article's Creative Commons license and your intended use is not permitted by statutory regulation or exceeds the permitted use, you will need to obtain permission directly from the copyright holder. To view a copy of this license, visit http://creativecommons.org/licenses/by/4.0/. 
with cell proliferation and migration ${ }^{11}$. Emerging evidence has linked other Rho GAPs to the development and progression of ovarian cancer ${ }^{12}$. However, the molecule mechanism and regulation of ARHGAP26 in ovarian cancer tumorigenesis is still unclear.

Ubiquitination is a posttranslational modification in which ubiquitin is attached to one or more lysine residues of cellular proteins through a series of enzymatic cascade reactions ${ }^{13}$. Similar to phosphorylation, ubiquitination alters the stability, conformation, or localization of the target proteins through reversible covalent modification, thereby regulating signal transduction, protein-protein interactions, gene transcription, and other biological processes ${ }^{14}$. Ubiquitination is catalyzed by a ubiquitinactivating enzyme E1, ubiquitin-conjugating enzyme E2, and ubiquitin ligase enzyme E3, the latter of which regulates the specificity of substrates in the ubiquitin proteasomal system. Smad ubiquitination regulatory factor 1 (SMURF1) is an E3 ubiquitin-protein ligase and increased SMURF1 expression has been observed in patients with ovarian cancer $^{15}$, promotes RhoA ubiquitination, and regulates cell growth and metastasis ${ }^{16}$. Nevertheless, the cellular function of SMURF1 and its role in regulation of ARHGAP26 in ovarian cancer remain largely unknown.

In this study, we report that ARHGAP26 is downregulated, whereas $\beta$-catenin and SMURF1 are upregulated in ovarian cancer patients. ARHGAP26 upregulation inhibited ovarian cancer cell proliferation, invasion, and migration in vitro and lung metastasis in vivo. ARHGAP26 downregulation promoted ovarian cancer cell invasion and migration by activating the $\beta$-catenin pathway. SMURF1 upregulation promoted ubiquitination of ARHGAP26 and induced ovarian cancer cell migration and invasion, which were inhibited by ARHGAP26 upregulation. These data suggest that SMURF1-mediated ubiquitination of ARHGAP26 may promote ovarian cancer cell invasion and migration via the $\beta$-catenin pathway.

\section{Materials and methods}

\section{Bioinformatics}

Gene expression data were obtained from The Cancer Genome Atlas (TCGA, https://tcga-data.nci.nih.gov/tcga/) for ovarian cancer projects, including 568 cases with tumor tissues and 8 cases with adjacent noncancerous tissues. Gene-set enrichment analysis (GSEA) was used to identify the pathways that were significantly enriched between patients with high and low ARHGAP26 expression.

\section{Tissue samples}

In total, 85 cases of tumor tissues and their corresponding adjacent noncancerous tissues were obtained from ovarian cancer patients in Bao'an Maternity and Child Health Hospital recruited from October 2012 to
March 2017. Human ovarian cancer and adjacent normal tissues were immediately snap-frozen in liquid nitrogen and stored at $-80^{\circ} \mathrm{C}$ until immunohistochemistry (IHC) was performed ${ }^{17}$. All of the patients provided signed informed consent. The medical ethics committee of Bao'an Maternity and Child Health Hospital approved the retrieval method for cancer specimens.

\section{Cell culture and transfection}

The human ovarian cancer cell lines OVCAR3, SKOV3, A2780, HEY, and CAOV3, and nonmalignant human ovarian surface epithelial cells IOSE80 were all purchased from the Shanghai Institute of Biochemistry and Cell Biology (Shanghai, China), and cultured in an incubator with $95 \%$ humidity and $5 \% \mathrm{CO}_{2}$ at $37^{\circ} \mathrm{C}$ in RPMI-1640 medium (HyClone, Logan, UT, USA) with $10 \%$ fetal bovine serum (Gibco Lab, Grand Island, NY, USA) and $1.0 \%$ penicillin-streptomycin solution (Solarbio, Beijing, China).

A2780 and HEY cells were cultured in six-well plates at $2 \times 10^{5}$ cells/well overnight and transduced with a lentiviral vector encoding ARHGAP26 (pLVX-Puro-ARHGAP26) or with a blank pLVX-Puro lentivirus as the negative control (blank vector). To silence ARHGAP26 expression, SKOV3 cells under the same culture conditions were transfected with ARHGAP26 small interfering RNA (siRNA) (siRNA-1, position 439-457, 5' GCUGGACAAGACCAACAAA- ${ }^{\prime}$; siRNA-2, position 1140-1158, 5' -CCAUCAGUCCCUACACCAU-3'; siRNA3, position 1213-1231, 5'-GCACUACUGUACAUAU CAA-3') or a control siRNA (siNC) using Lipofectamine 2000 (Invitrogen, Carlsbad, CA, USA) according to the manufacturer's instructions and incubated for $6 \mathrm{~h}$ at $37^{\circ} \mathrm{C}$. After being incubated in complete RPMI-1640 medium for $48 \mathrm{~h}$, SKOV 3 cells were treated with or without $200 \mathrm{ng} / \mathrm{mL}$ DKK1. Otherwise, SKOV3 cells were transduced with pLVX-Puro-WWP1, pLVX-Puro-CBL, pLVX-PuroNEDD4, pLVX-Puro-MDM2, pLVX-Puro-SMURF1, or pLVX-Puro-ARHGAP26 alone or in combination with one another.

\section{CCK-8 assay}

A Cell Counting Kit (CCK-8) assay was used to measure cell proliferation. Cells were cultured in 96-well plates at $3 \times 10^{3}$ cells/well for 24,48 , and $72 \mathrm{~h}$. Then, each well was filled with $10 \mu \mathrm{L}$ of CCK-8 solution and incubation was continued at $37^{\circ} \mathrm{C}$ for an additional $1 \mathrm{~h}$. The absorbance of each well was determined at $450 \mathrm{~nm}$.

\section{Transwell assay}

Cells were serum-starved in serum-free RPMI-1640 medium for $24 \mathrm{~h}$ and cell suspensions $\left(9 \times 10^{4} /\right.$ well $)$ were plated in Transwell chambers with (invasion assay) or without (migration assay) Matrigel coating. Then, $700 \mu \mathrm{L}$ 
of RPMI-1640 medium supplemented with $10 \%$ fetal bovine serum was added to the lower chamber. After $48 \mathrm{~h}$ of incubation, the cells were fixed, stained, photographed, and counted under a light microscope (Olympus Corporation, Tokyo, Japan) at $\times 200$ magnification.

\section{Quantitative real-time PCR}

Total RNA was extracted from ovarian cancer tissues and cell lines using Trizol reagent (Invitrogen) according to the manufacturer's protocol and reverse-transcribed with a RevertAid First Strand cDNA Synthesis Kit (Thermo Fisher, Waltham, MA, USA). Quantitative realtime PCR was carried out using SYBR green PCR Master Mix (Fermentas, Vilnius, Lithuania) on an ABI7500 system following the manufacturer's instructions. The forward and reverse primers used for quantitative real-time PCR amplification were as follows: ARHGAP26 (5'-AATTCCAGCAGCAGCTTAC- ${ }^{\prime}$ and 5'-TTCAGCTTTGCAGGCATAC-3'); SMURF1 (5'-CTG GCAAGCGGTGGAGAC-3' and 5'-CCGGTTAAAGCAGGTATGGG-3'); CBL (5'-CATCTGCCAATGCCAT TTATTC-3' and $5^{\prime}$-GCTATCAATCTGCTGGTCGC-3'); NEDD4 (5'-AGTTTGTCACTGGCACATCTCG-3' and 5'-CAGCTTTTCAGGAGTACCCCAC-3'); MDM2 (5'-G GGCTTTGATGTTCCTGATTG- $3^{\prime}$ and $5^{\prime}$-TTCTTTGT CTTGGGTTTCTTCC-3'); WWP1 (5'-ATAATGCGT CTGTCACGGGTAC-3' and 5'-GCTGTCTTGATTTGG CTGCTTC-3'); GAPDH (5'-AATCCCATCACCATCT TC-3' and $5^{\prime}$-AGGCTGTTGTCATACTTC-3'). The relative abundance of genes was quantified using the comparative $2^{-\Delta \Delta C t}$ method with GAPDH as the internal control.

\section{Western blotting}

Cell lysates were prepared as previously described ${ }^{17}$. An equal amount of protein was incubated with glutathione $S$-transferase-rhotekin (Upstate Biotechnologies, Inc., Lake Placid, NY, USA) for $45 \mathrm{~min}$ at $4{ }^{\circ} \mathrm{C}$ to collect the active form of RhoA (GTP-RhoA). Immunoblotting was performed with antibodies against ARHGAP26 (Abcam, Cambridge, MA, USA), RhoA (Santa Cruz Biotechnology, Santa Cruz, CA, USA), MMP2 (Abcam), MMP7 (Abcam), SMURF1 (Abcam), CBL (Abcam), NEDD4 (Abcam), MDM2 (Abcam), WWP1 (Abcam), VEGF (Affinity, Cincinnati, OH, USA), $\beta$-catenin (Cell Signaling Technology, Danvers, MA, USA), GAPDH (Cell Signaling Technology), and horseradish peroxidase-labeled goat anti-rabbit IgG (Beyotime Biotechnology, Shanghai, China). Proteins on membranes were detected using an ECL system (Amersham Biosciences, Piscataway, NJ, USA).

\section{Co-immunoprecipitation}

Whole-cell lysates obtained by centrifugation were incubated with $2 \mu \mathrm{g}$ anti-ARHGAP26, anti-SMURF1 or normal IgG antibody, and protein G-Agarose beads (Roche Diagnostics Ltd, Shanghai, China) at $4{ }^{\circ} \mathrm{C}$ overnight. The immunocomplexes were then separated via sodium dodecyl-sulfate polyacrylamide gel electrophoresis (SDS-PAGE), and blottings were probed with the indicated antibody.

\section{Ubiquitination assay}

SKOV3 cells were transduced with pLVX-PuroSMURF1 or blank lentivirus (blank vector). After $48 \mathrm{~h}$, cell lysates were subjected to immunoprecipitation with anti-ARHGAP26 or normal IgG antibody for $4 \mathrm{~h}$ at $4{ }^{\circ} \mathrm{C}$. After incubation with protein G-agarose beads at $4{ }^{\circ} \mathrm{C}$ for $1 \mathrm{~h}$, the immunocomplexes were separated via SDS-PAGE and subjected to western blotting analysis with anti-ubiquitin antibody.

\section{Animal experiments}

A total of $1 \times 10^{6}$ A2780 or HEY cells transduced with pLVX-Puro-ARHGAP26 or blank vector were collected, resuspended in $100 \mu \mathrm{L}$ phosphate-buffered saline, and then intravenously injected through the tail vein into 5-6week-old female BALB/c nude mice (weight: 16-18 g; 6 per group). Mice were killed 21 days after injection and the lung tissues were removed from the xenograft mice and stained with hematoxylin and eosin as previously described $^{18}$. Animal experiments were approved by the Bao'an Maternity and Child Health Hospital institutional ethical committee and performed according to the legal requirements.

\section{Statistical analysis}

Each experiment was performed in triplicate. The data were analyzed using GraphPad Prism 5.0 software (GraphPad Software, Inc., La Jolla, CA, USA) and are presented as the mean $\pm \mathrm{SD}$. Differences between groups were calculated using one-way or two-way analysis of variance. All statistical tests were two-sided with an $\alpha$ level of 0.05 .

\section{Results}

\section{Decreased ARHGAP26 expression in ovarian cancer tissues}

We analyzed the expression levels of ARHGAP26 in ovarian cancer tissues using TCGA and our independent hospital databases. The results demonstrated downregulated ARHGAP26 mRNA expression in ovarian cancer tissues compared with adjacent noncancerous ovarian tissues from TCGA (Fig. 1a) and independent hospital cohorts (Fig. 1b).

We also examined ARHGAP26 protein expression in ovarian cancer tissues via IHC analysis, which showed that ARHGAP26 protein expression was markedly decreased in tumor tissues; $57.6 \%$ (49/85) of ovarian cancer tissues displayed negative or low expression of 

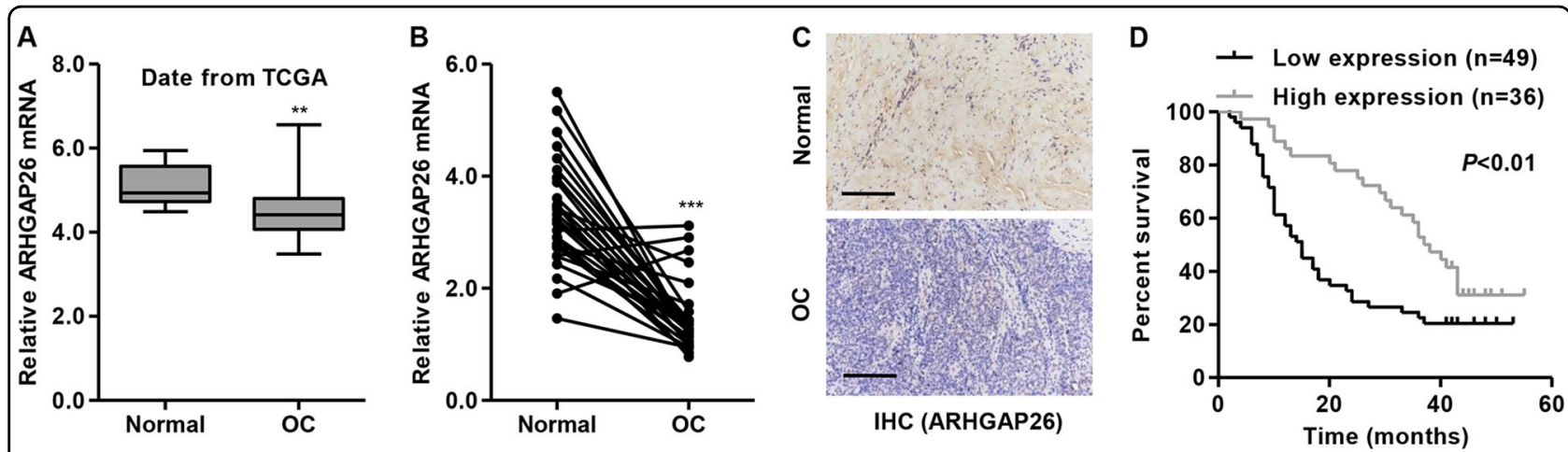

Fig. 1 ARHGAP26 expression was decreased in ovarian cancer tissues. ARHGAP26 mRNA expression in ovarian cancer tissues from TCGA (a) and our independent hospital (b) cohorts was detected. ARHGAP26 protein expression in our independent hospital (c) cohorts was detected via immunohistochemistry $\left(\mathrm{IHC}\right.$ ). Scale bars: $100 \mu \mathrm{m}$. d Overall survival of 85 patients with ovarian cancer from the hospital cohort was detected. ${ }^{* *} P<$ 0.01 and ${ }^{* *} P<0.001$ compared with normal tissues. OC, ovarian cancer; normal, adjacent noncancerous tissues

ARHGAP26 ( $<25 \%$ of tumor cells were positively stained) (Fig. 1c). The correlation between ARHGAP26 expression and the prognosis of patients with ovarian cancer was analyzed with a log-rank (Mantel-Cox) test. The results indicated that the overall survival of patients with lower ARHGAP26 expression was significantly shorter than that of patients with higher ARHGAP26 expression (Fig. 1d), suggesting that decreased ARHGAP26 expression may contribute to poor survival.

\section{ARHGAP26 overexpression inhibits ovarian cancer cell proliferation}

To further examine the role of ARHGAP26 in ovarian cancer tumorigenesis in vitro, ARHGAP26 expression in five ovarian cancer cell lines (OVCAR3, SKOV3, A2780, HEY, and CAOV3) was assessed. As shown in Fig. 2a, b, decreased ARHGAP26 expression was observed in ovarian cancer cell lines compared with that in IOSE80 cells, with the two lowest expression levels detected in A2780 and HEY cells, and the highest expression detected in SKOV3 cells. Therefore, we stably overexpressed ARHGAP26 in A2780 and HEY cells, and its expression in cells transduced with ARHGAP26 overexpression lentivirus was significantly elevated compared with control cells or cells transduced with blank vector (Fig. 2c, d). Moreover, CCK8 assays demonstrated that the proliferation of A2780 and HEY cells overexpressing ARHGAP26 was significantly suppressed at 48 and $72 \mathrm{~h}$ compared with control cells or cells transduced with blank vector (Fig. 2e, f).

\section{ARHGAP26 overexpression inhibits ovarian cancer cell invasion and migration}

To determine whether ARHGAP26 affects ovarian cancer cell migration and invasion, Transwell assays were performed. Our data showed that the migration and invasion of A2780 cells overexpressing ARHGAP26 were significantly suppressed by $51.4 \%$ and $52.6 \%$ compared with control cells (Fig. 3a, b). Similarly, the migration and invasion of HEY cells overexpressing ARHGAP26 were significantly suppressed by $52.3 \%$ and $53.1 \%$ compared with control cells (Fig. 3c, d). In view of the GSEA data showing that high ARHGAP26 expression is negatively correlated with $\mathrm{Wnt} / \beta$-catenin signaling (Supplementary Fig. S1), the expression of $\beta$-catenin and the downstream effectors VEGF, MMP2, and MMP7, as well as total RhoA and GTP-RhoA, were examined via western blotting. Our results showed that ARHGAP26 overexpression in A2780 and HEY cells significantly inhibited the expression of these proteins, except RhoA, compared with control cells or cells transduced with blank vector (Fig. 3e, g).

Next, we investigated the effect of ARHGAP26 on tumor metastasis in vivo. Tumor metastasis was examined after A2780 or HEY cells stably expressing ARHGAP26 or blank vector were intravenously injected into the tail vein of nude mice. Compared with the mice injected with A2780 or HEY cells transduced with blank vector, less cell metastasis was observed in the lung tissues of nude mice 21 days after injection with A2780 or HEY cells stably expressing ARHGAP26 (Fig. 3h).

\section{Silencing of ARHGAP26 promotes ovarian cancer cell proliferation, migration, and invasion}

To further explore the role of ARHGAP26 in ovarian tumorigenesis, ARHGAP26 was silenced using siRNA in SKOV3 cells. ARHGAP26 expression in SKOV3 cells transfected with siRNA-1, siRNA-2, or siRNA-3 was significantly decreased by $75.6 \%, 87.1 \%$, and $92.6 \%$ at the mRNA level and by $45.3 \%, 69.7 \%$, and $83.5 \%$ at the protein level compared with control cells, respectively (Fig. 4a, b). Therefore, siRNA-2 and siRNA-3 were used in our following experiments. SKOV3 cells transfected with siRNA2 or siRNA-3 showed increased cell proliferation, migration, and invasion compared with control cells or cells transfected with siNC (Fig. 4c, e). In addition, silencing of 

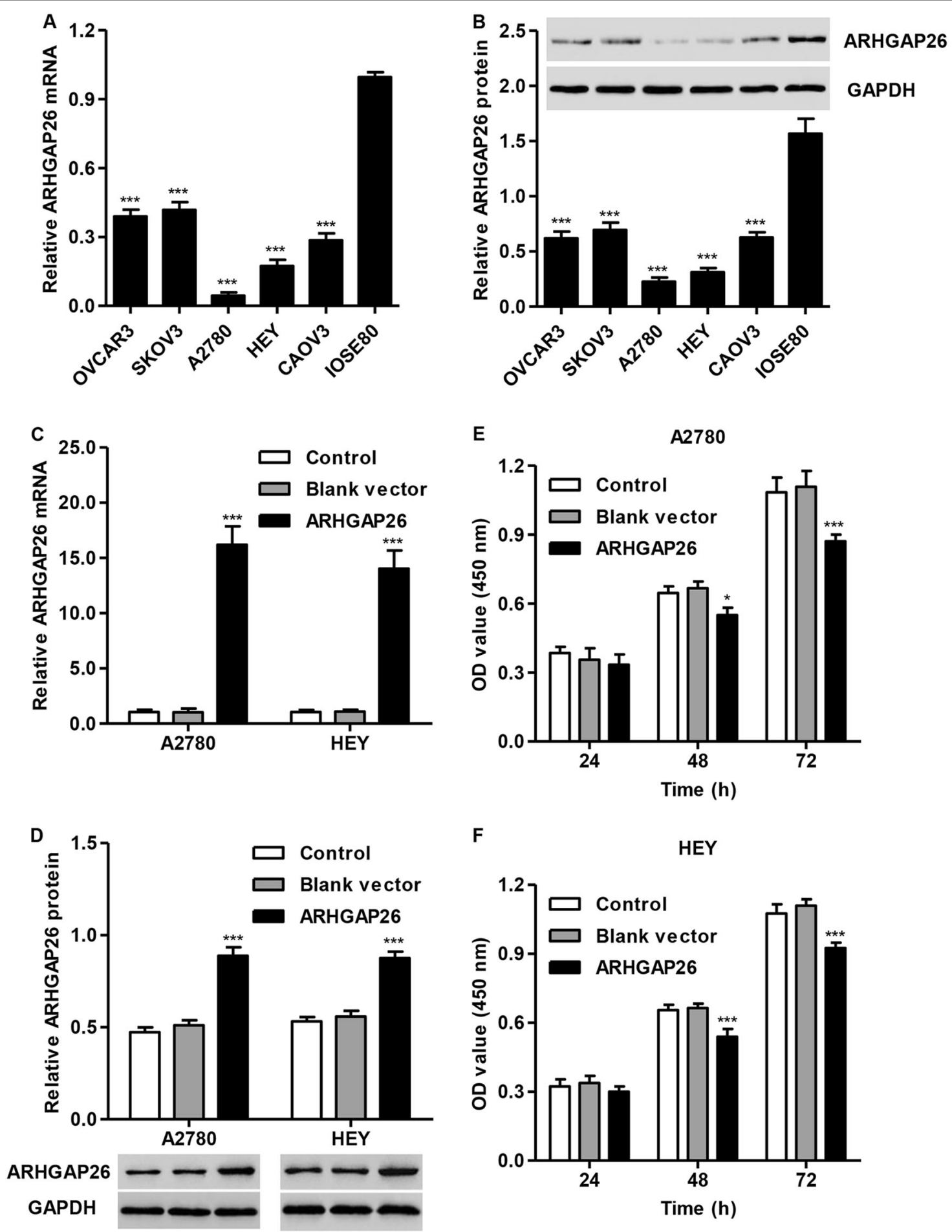

$\mathbf{F}$

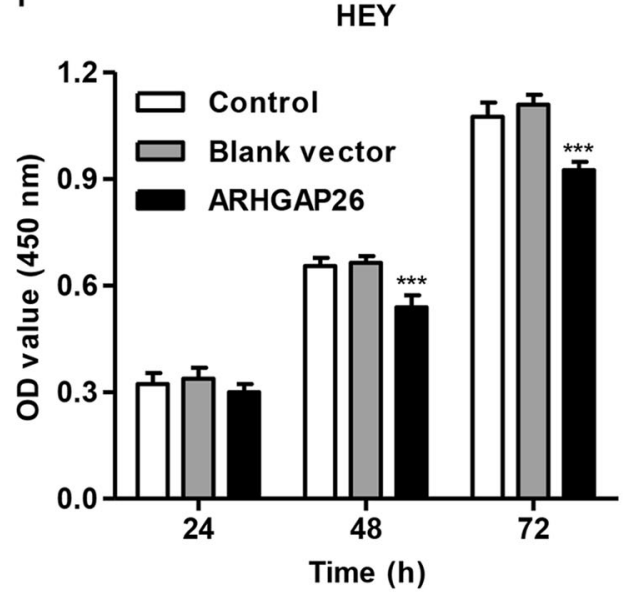

Fig. 2 ARHGAP26 upregulation inhibited the proliferation of A2780 and HEY cells. a, b ARHGAP26 expression in five ovarian cancer cell lines and the nonmalignant human ovarian surface epithelial cell line IOSE80 was detected. A2780 and HEY cells were transduced with ARHGAP26 expression lentivirus or control lentivirus (blank vector). ARHGAP26 expression (c, d) and cell proliferation (e, $\mathbf{f}$ ) was detected via real-time PCR, western blotting, and CCK-8 assays. ${ }^{*} P<0.05$ and ${ }^{* *} P<0.001$ compared with IOSE80 or control cells 


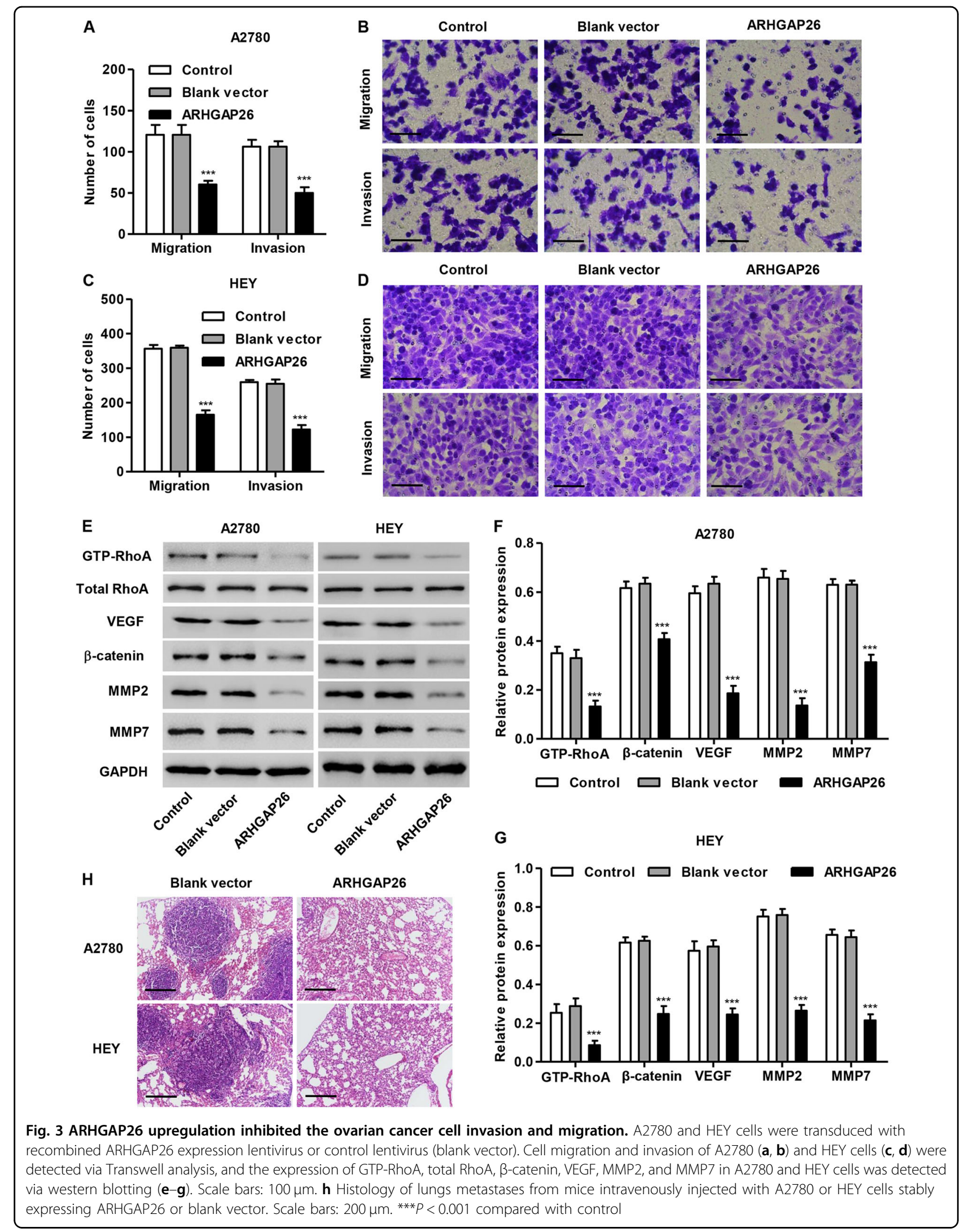




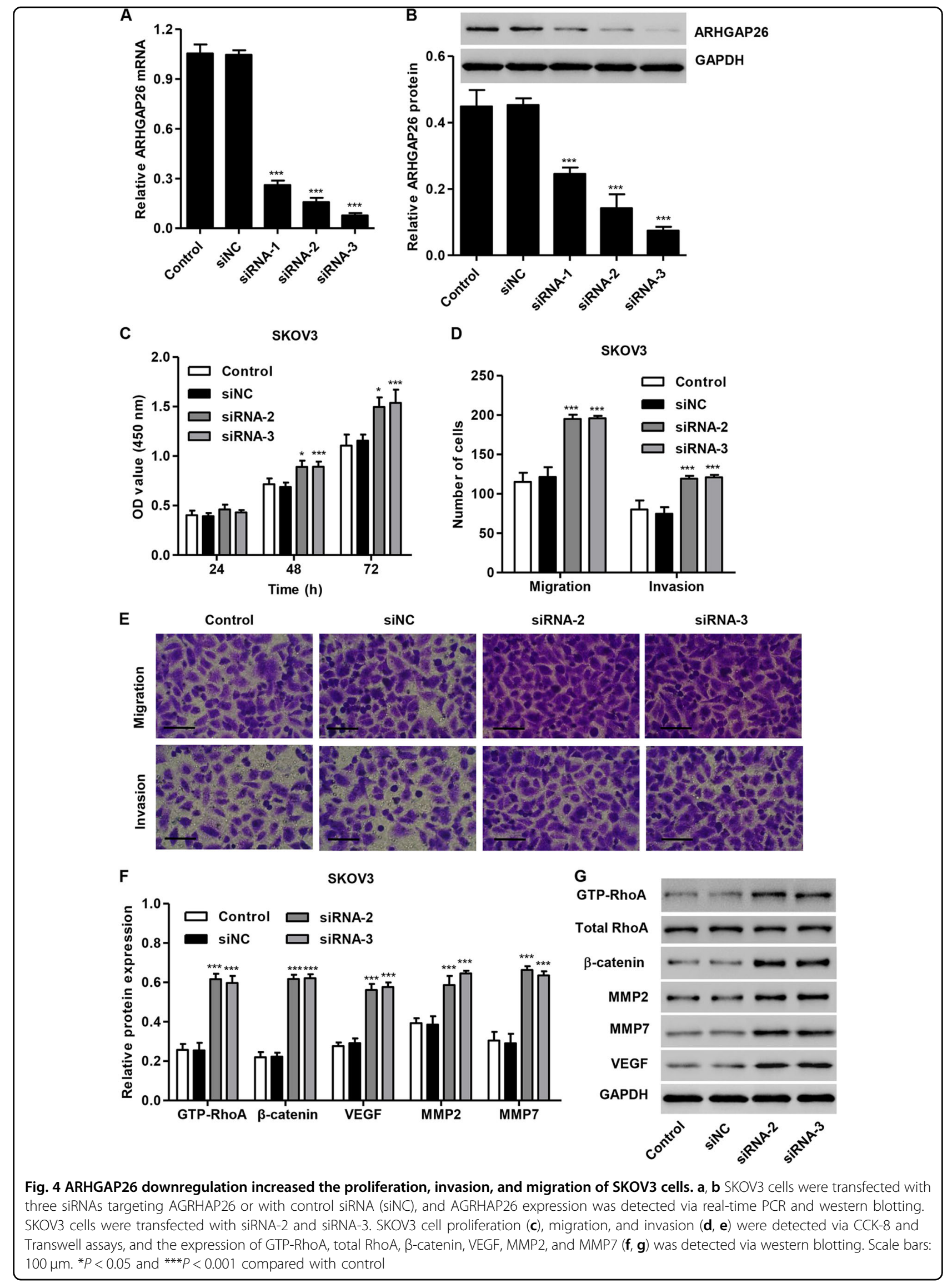




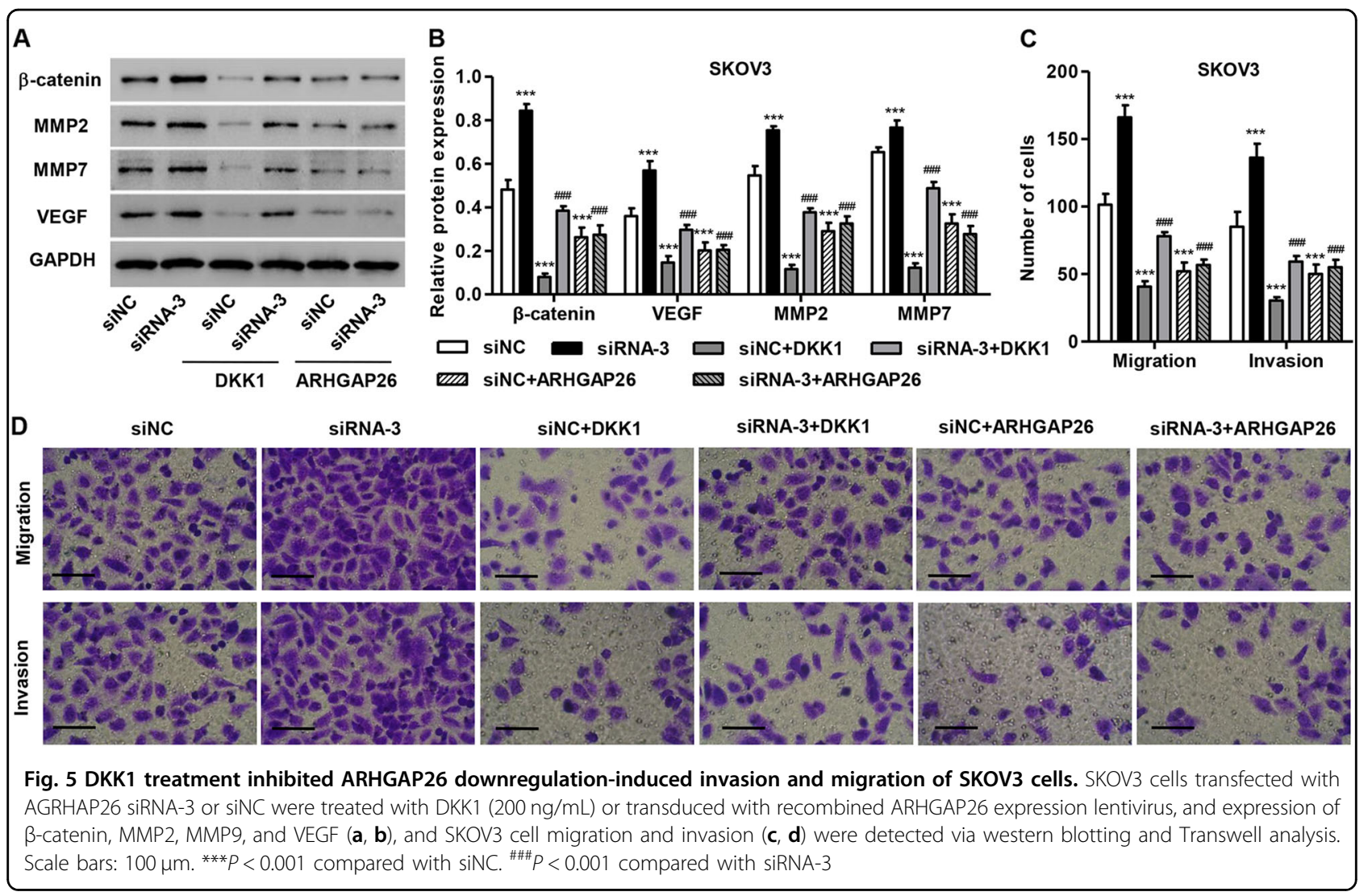

ARHGAP26 significantly increased the expression level of GTP-RhoA, $\beta$-catenin, VEGF, MMP2, and MMP7 in SKOV3 cells (Fig. 4f, g). These data indicated that ARHGAP26 may be a potential regulator of tumor migration and invasion in ovarian cancer.

\section{DKK1 inhibits ARHGAP26 silencing-induced ovarian cancer cell invasion and migration}

Next, we tried to explore the effects of $\beta$-catenin signaling on ARHGAP26-mediated ovarian tumorigenesis and an antagonist of the $\beta$-catenin pathway (DKK1) was introduced in SKOV3 cells. As expected, DKK1 treatment significantly reduced $\beta$-catenin expression in SKOV3 cells transfected with siNC or siRNA-3 (Fig. 5a, b). Importantly, DKK1 treatment significantly inhibited the migration and invasion of SKOV3 cells with silencing of ARHGAP26 (Fig. 5c, d). These results suggested that ARHGAP26 regulates ovarian cancer cell invasion and migration through $\beta$-catenin signaling.

\section{SMURF1 promotes ovarian cancer cell migration and invasion by inhibiting ARHGAP26}

According to the UbiBrowser database (http:// ubibrowser.ncpsb.org/), ARHGAP26 is predicted to be ubiquitinated by multiple E3 ubiquitin ligases, such as SMURF1, CBL, NEDD4, MDM2, and WWP1, which have been found to be dysregulated in ovarian cancer and associated with ovarian cancer cell proliferation and metastasis $^{15,19-22}$, with a high and middle confidence interaction. Therefore, the associations between these E3 ubiquitin ligases and ARGHAP26 were further confirmed. We stably overexpressed SMURF1, CBL, NEDD4, MDM2, or WWP1 in SKOV3 cells and the expression of these proteins was significantly increased compared with that in control cells or cells treated with blank vector (Supplementary Fig. S2). SMURF1, CBL, NEDD4, or MDM2 overexpression significantly reduced the expression of ARHGAP26, with the lowest expression detected in SKOV3 cells with SMURF1 overexpression (Supplementary Fig. S3). Furthermore, SMURF1 expression levels in ovarian cancer cell lines were also measured. As shown in Fig. 6a, b, increased SMURF1 expression was observed in ovarian cancer cell lines compared with that in IOSE80 cells, with the lowest expression detected in SKOV3 cells. SMURF1 coimmunoprecipitated with ARHGAP26 (Fig. 6c). Reciprocal immunoprecipitation with ARHGAP26 antibodies also brought down SMURF1, suggesting an interaction between SMURF1 and ARHGAP26 in SKOV3 cells. In addition, the decrease in the ARHGAP26 level could be reversed by the addition of the proteasome inhibitor MG132, suggesting that SMURF1 regulates ARHGAP26 levels in a proteasome-dependent manner 


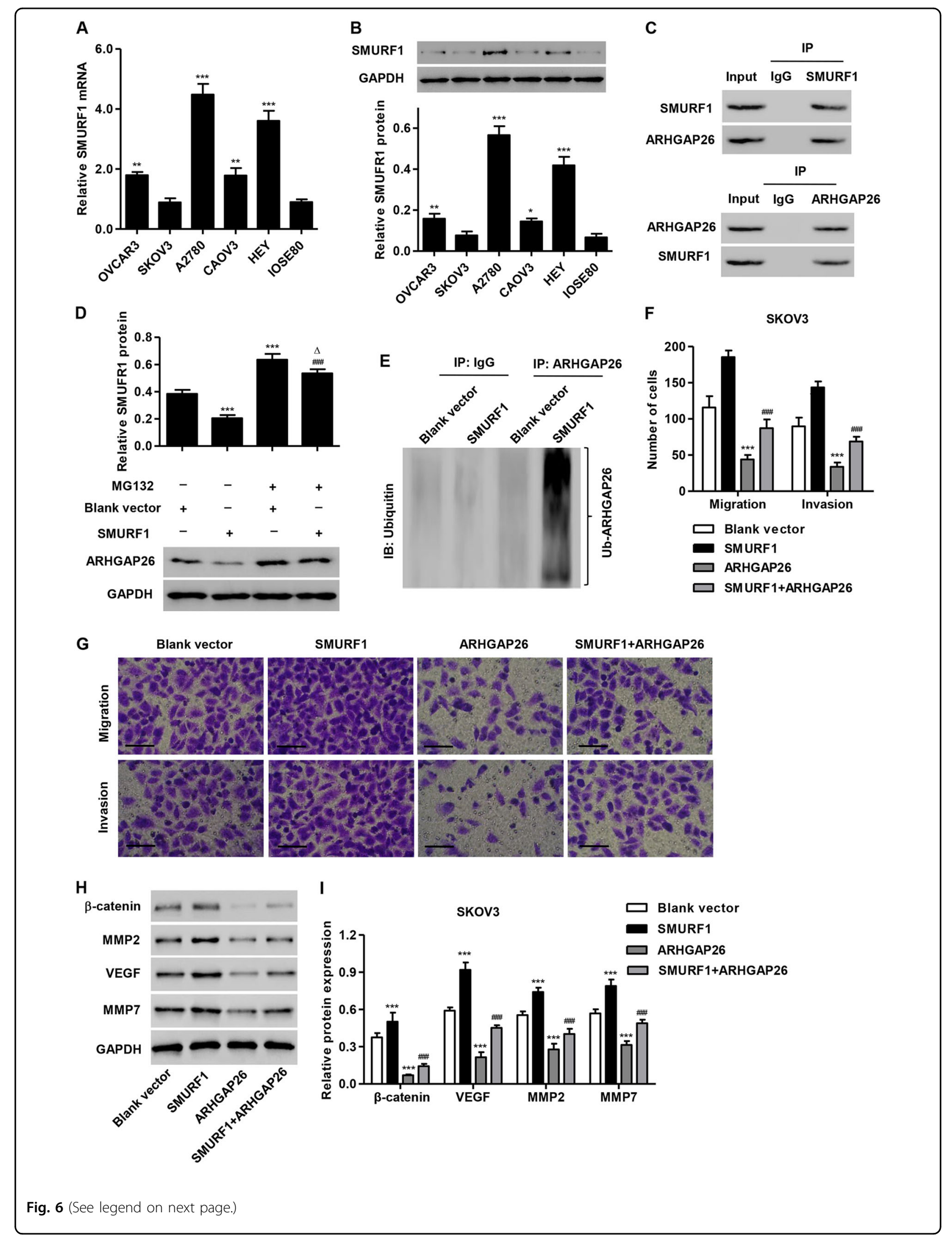


(see figure on previous page)

Fig. 6 SMURF1 promotes ovarian cancer cell migration and invasion by inhibiting ARHGAP26. a, b SMURF1 expression in five ovarian cancer cell lines and the nonmalignant human ovarian surface epithelial cell line IOSE80 was detected. c SKOV3 cell lysates were subjected to immunoprecipitation with control IgG, or anti-SMURF1 or anti-ARHGAP26 antibody. The immunoprecipitates were then blotted with anti-SMURF1 or anti-ARHGAP26 antibody. d SKOV3 cells transduced with recombined pLVX-Puro-SMURF1 or control lentivirus (blank vector) were treated with MG132 $(50 \mu \mathrm{M})$ for $4 \mathrm{~h}$, and the expression of ARHGAP26 was determined by western blotting. e SKOV3 cells were transduced with recombined pLVX-Puro-SMURF1 or control lentivirus (blank vector). After $48 \mathrm{~h}$, the cells were collected and AGRHAP26 was immunoprecipitated and immunoblotted with control lgG, or anti-ARHGAP26 or anti-ubiquitin antibody. SKOV3 cells were transduced with recombined pLVX-Puro-SMURF1, pLVX-Puro-ARHGAP26, or control lentivirus (blank vector), and cell migration, invasion (f, $\mathbf{g}$ ), and expression of $\beta$-catenin, VEGF, MMP2, and MMP7 (h, i) were detected using Transwell and western blotting assays. Scale bars: $100 \mu \mathrm{m}$. ${ }^{*} P<0.05$, ${ }^{* *} P<0.01$, and ${ }^{* * *} P<0.001$ compared with IOSE80 cells or cells transduced with blank vector. ${ }^{\# \# \#} P<0.001$ compared with SMURF1. ${ }^{\Delta} P<0.05$ compared with blank vector plus MG132

(Fig. 6d). Moreover, SMURF1 overexpression in SKOV3 cells significantly induced ubiquitination of ARHGAP26 (Fig. 6e). Importantly, SMURF1 overexpression significantly promoted the migration and invasion of SKOV3 cells (Fig. 6f, g), and increased the expression of $\beta$-catenin, VEGF, MMP2, and MMP7 (Fig. 6h, i), and these effects were reversed by ARHGAP26 overexpression.

\section{Expression of $\beta$-catenin and SMURF1, and correlation analyses in ovarian cancer tissues}

We analyzed the mRNA expression levels of $\beta$-catenin and SMURF1 in ovarian cancer tissues and performed a Pearson's correlation analysis between ARHGAP26 and $\beta$-catenin or SMURF1 and $\beta$-catenin. The results demonstrated upregulated mRNA expression of $\beta$-catenin and SMURF1 in ovarian cancer tissues compared with corresponding adjacent noncancerous ovarian tissues from our independent hospital cohort (Fig. 7a, b). ARHGAP26 mRNA expression was negatively correlated with the mRNA expression of $\beta$-catenin in ovarian cancer tissues (Fig. 7c), whereas SMURF1 mRNA expression was positively correlated with the mRNA expression of $\beta$-catenin in ovarian cancer tissues (Fig. 7d).

\section{Discussion}

Ovarian cancer possesses great potential for growth and metastasis, resulting in higher morbidity, mortality, and health-care $\operatorname{costs}^{23}$. Herein, the present study assessed ARHGAP26 expression and its underlying mechanism in regulation of ovarian cancer cell proliferation, invasion, and migration. Our results provided evidence, suggesting that ARHGAP26 inhibits ovarian cancer cell invasion and migration in vivo and in vitro, and SMURF1-mediated ARHGAP26 ubiquitination may promote ovarian cancer cell invasion and migration via the $\beta$-catenin signaling pathway.

Previous studies showed significantly downregulated ARHGAP26 expression in glioblastoma and myeloid malignancies ${ }^{9,11}$. In the current study, we clarified for the first time that ARHGAP26 expression is decreased in ovarian cancer tissues compared with adjacent noncancerous ovarian tissues from TCGA and our independent hospital databases, and in ovarian cancer cell lines compared with nonmalignant human ovarian surface epithelial cells, and found that ovarian cancer patients with high ARHGAP26 expression tended to have a poor prognosis, suggesting that ARHGAP26 may act as a tumor suppressor involved in ovarian cancer development. ARHGAP26 disruption induced a dramatic loss of an epithelial cellular phenotype and increased gastric cancer cell invasion and migration ${ }^{24}$. Loss of ARHGAP26 activity also dramatically increased the invasion of SW480 colorectal cancer cells ${ }^{25}$. Here we demonstrated a similar role of ARHGAP26 in regulation of cell proliferation, invasion, and migration in ovarian cancer. ARHGAP26 overexpression inhibited ovarian cancer cell proliferation, migration, and invasion, and lung metastasis in xenograft nude mice bearing ovarian cancer, whereas ARHGAP26 silencing promoted these behaviors. Therefore, we came to the conclusion that ARHGAP26 is of great importance in the tumorigenesis of ovarian cancer.

Other remarkable findings of the present study were that ARHGAP26 overexpression inhibited the expression of $\beta$-catenin, VEGF, MMP2, and MMP7, whereas ARHGAP26 silencing promoted the expression of these proteins. These findings are consistent with GSEA data that shows negative regulation between ARHGAP26 and these proteins. $\beta$-catenin, the core of the classical Wnt signaling pathway, is highly expressed in many malignant tumors and associated with chemotherapy resistance, recurrence, and prognosis in ovarian cancer ${ }^{26,27}$. Moreover, $\beta$-catenin can increase the transcription of downstream $\beta$-catenin signaling pathway-related genes, including VEGF, MMP2, and MMP7, thereby leading to tumor cell proliferation and metastasis ${ }^{28,29}$. In the present study, ovarian cancer cells treated with DDK1, an antagonist of the $\beta$-catenin pathway, demonstrated decreased cell migration and invasion induced by ARHGAP26 silencing, indicating that ARHGAP26 regulates ovarian cancer cell invasion and migration by regulating VEGF, MMP2, and MMP7 expression through the $\beta$-catenin signaling pathway. ARHGAP26 is a GTPaseactivating protein that was found to be inversely correlated with the expression of the GTP-bound form of RhoA 

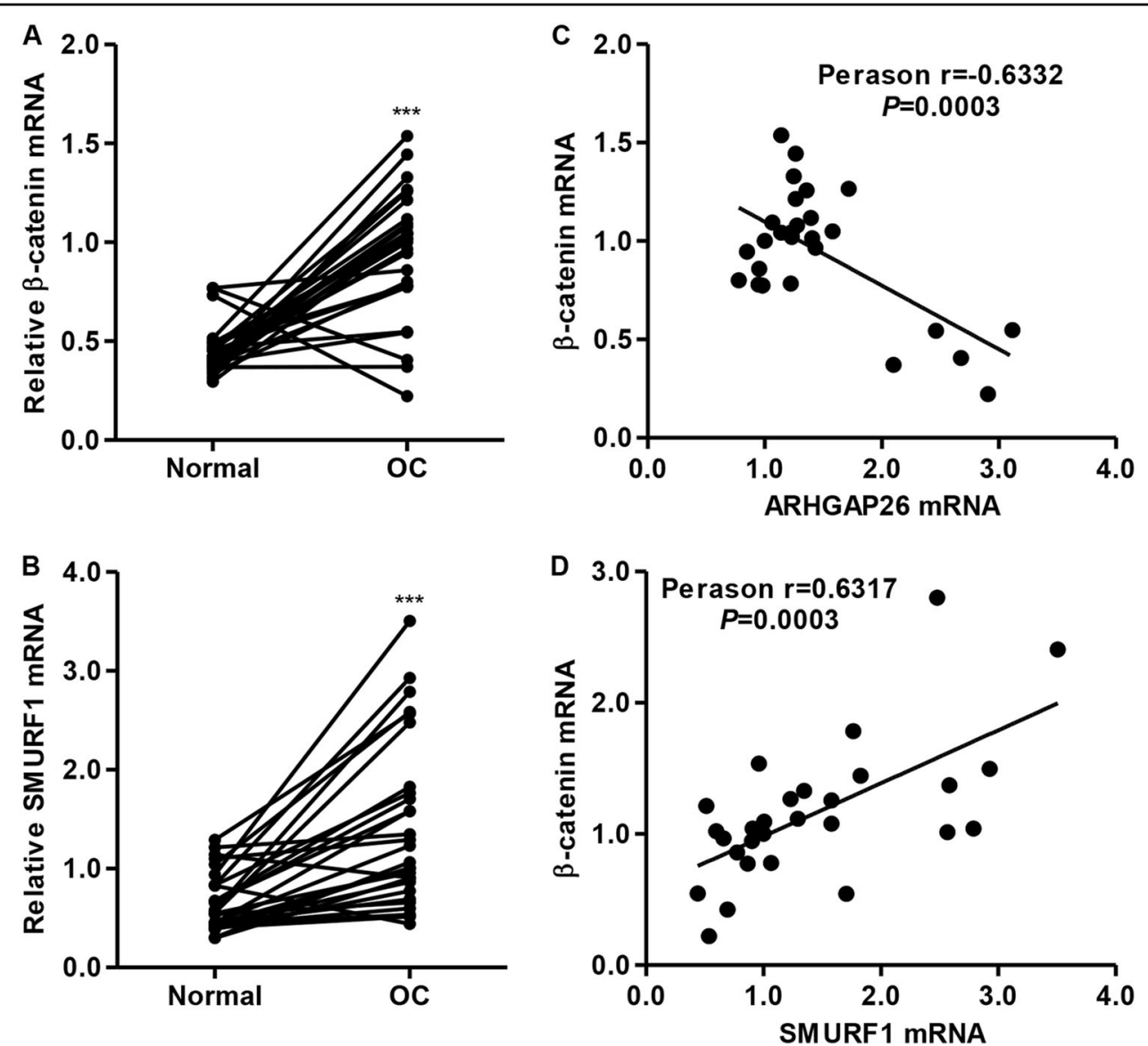

Fig. 7 Expression of $\beta$-catenin and SMURF1 and correlation analysis in ovarian cancer tissues. $\mathbf{a}, \mathbf{b} \beta$-Catenin and SMURF1 mRNA expression in ovarian cancer tissues and adjacent normal ovarian tissues from our independent hospital database was detected using real-time PCR. $\mathbf{c}$, $\mathbf{d}$ Pearson's correlation scatter plots for ovarian cancer tissues $(n=28)$. ${ }^{* *} P<0.001$ compared with normal. OC, ovarian cancer; normal, adjacent noncancerous tissues

(GTP-RhoA), the active form of RhoA. Moreover, a previous study reported that activated RhoA contributes to $\beta$-catenin accumulation, leading to an increase in cell migration $^{30}$, which suggests that ARHGAP26 may regulate $\beta$-catenin expression through RhoA. However, inactivation of RhoA can contribute to colorectal cancer progression and metastasis through activation of $\mathrm{Wnt} / \beta$-catenin signaling ${ }^{31}$. Therefore, the discrepancy between RhoA activity and $\beta$-catenin accumulation may be attributed to differences in cell types/tissues and it warrants further study. Moreover, $\beta$-catenin mRNA expression was found to be increased in ovarian cancer tissues and negatively correlated with ARHGAP26 mRNA expression.

Whether ARHGAP26 can be regulated in ovarian cancer remains an unanswered question. According to the UbiBrowser database (http://ubibrowser.ncpsb.org/), ARHGAP26 is predicted to be ubiquitinated by multiple E3 ubiquitin ligases, such as SMURF1, CBL, NEDD4, MDM2, and WWP1, which have been found to be dysregulated in ovarian cancer and associated with ovarian cancer cell proliferation and metastasis ${ }^{15,19-22}$. In this study, compared with other E3 ubiquitin ligases, the decrease in ARHGAP26 protein expression in cells with SMURF1 overexpression was the most significant and SMURF1 overexpression promoted ARHGAP26 ubiquitination and ovarian cancer cell invasion and migration, along with an increase in $\beta$-catenin, VEGF, MMP2, and MMP7 expression. Similarly, SMURF1 has been found to be increased in ovarian cancer patients and be associated with cell migration and invasion in ovarian cancer ${ }^{15}$. ARHGAP26 overexpression inhibited SMURF1-mediated cell migration, invasion, and $\beta$-catenin signaling in ovarian cancer, thereby suggesting that SMURF1-mediated ARHGAP26 ubiquitination may promote ovarian cancer cell invasion and migration via the $\beta$-catenin signaling pathway. Moreover, SMURF1 mRNA expression was positively correlated with $\beta$-catenin mRNA expression in ovarian cancer tissues. 
In conclusion, the present study preliminarily provides insights into the anticancer effect of ARHGAP26 in ovarian cancer cells. ARHGAP26 overexpression inhibits the proliferation, migration, and invasion of ovarian cancer cells. SMURF1-mediated ubiquitination of ARHGAP26 may promote ovarian cancer cell invasion and migration through the $\beta$-catenin signaling pathway. Therefore, ARHGAP26 may be a promising novel therapeutic target for ovarian cancer treatment.

\section{Acknowledgements}

This study was supported by the National Natural Science Foundation of China (81460394), the Shenzhen Science and Technology Innovation Committee Foundation (JCYJ20160429172357927), and the Sanming Project of Medicine in Shenzhen (SZSM201406007).

\section{Author details}

${ }^{1}$ Department of Obstetrics and Gynecology, Bao'an Maternity and Child Health Hospital, Jinan University, Shenzhen 518100, China. ${ }^{2}$ Maternal-Fetal Medicine Institute, Bao'an Maternity and Child Health Hospital, Jinan University, Shenzhen 518100, China. ${ }^{3}$ Shenzhen children's Hospital, Shenzhen 518000, China

\section{Author contributions}

X.C., H.L., and Y.L. designed the study. S.C., Y.G., and S.H. performed the experiments. Y.L., H.L., and Y.Z. collected, analyzed, and interpreted the data. X. C., S.C., and Y.Z. prepared the manuscript. All authors read and approved the final manuscript.

\section{Conflict of interest}

The authors declare that they have no conflict of interest.

\section{Publisher's note}

Springer Nature remains neutral with regard to jurisdictional claims in published maps and institutional affiliations.

Supplementary information accompanies this paper at https://doi.org/ 10.1038/s12276-019-0236-0.

Received: 11 September 2018 Revised: 6 December 2018 Accepted: 24 December 2018.

Published online: 19 April 2019

\section{References}

1. Nakata, S., Phillips, E. \& Goidts, V. Emerging role for leucine-rich repeat-containing G-protein-coupled receptors LGR5 and LGR4 in cancer stem cells. Cancer Manag. Res. 6, 171-180 (2014)

2. Paik, D. Y. et al. Stem-like epithelial cells are concentrated in the distal end of the fallopian tube: a site for injury and serous cancer initiation. Stem Cells $\mathbf{3 0}$ 2487-2497 (2012).

3. Vargas, A. N. Natural history of ovarian cancer. Ecancermedicalscience $\mathbf{8}, 465$ (2014).

4. Siegel, R. L., Miller, K. D. \& Jemal, A. Cancer statistics, 2018. CA Cancer J. Clin. 68 7-30 (2018)

5. Hasebe, T., Fujimoto, K., Kajita, M. \& Ishizuya-Oka, A. Thyroid hormone activates Wnt/beta-catenin signaling involved in adult epithelial development during intestinal remodeling in Xenopus laevis. Cell Tissue Res. 365, 309-318 (2016).

6. Ohta, K. et al. Graf1 controls the growth of human parainfluenza virus type 2 through inactivation of RhoA signaling. J. Virol. 90, 9394-9405 (2016).
7. Chen, $X$. et al. Supervillin promotes epithelial-mesenchymal transition and metastasis of hepatocellular carcinoma in hypoxia via activation of the RhoA ROCK-ERK/p38 pathway. J. Exp. Clin. Cancer Res. 37, 128 (2018).

8. Brauer, P. R. et al. Kruppel-like factor 4 mediates cellular migration and invasion by altering RhoA activity. Cell. Commun. Adhes. 24, 1-10 (2018).

9. Aly, R. M. \& Ghazy, H. F. High expression of GTPase regulator associated with the focal adhesion kinase (GRAF) is a favorable prognostic factor in acute myeloid leukemia. Blood Cells Mol. Dis. 53, 185-158 (2014).

10. Borkhardt, A. et al. The human GRAF gene is fused to MLL in a unique $t(5 ; 11)$ (q31;q23) and both alleles are disrupted in three cases of myelodysplastic syndrome/acute myeloid leukemia with a deletion 5q. Proc. Natl Acad. Sci. USA 97, 9168-9173 (2000).

11. Zohrabian, V. M. et al. Gene expression profiling of metastatic brain cancer. Oncol. Rep. 18, 321-328 (2007).

12. Luo, N. et al. ARHGAP10, downregulated in ovarian cancer, suppresses tumorigenicity of ovarian cancer cells. Cell Death Dis. 7, e2157 (2016).

13. Finley, D. \& Chau, V. Ubiquitination. Annu. Rev. Cell. Biol. 7, 25-69 (1991).

14. Fuchs, S. Y. Ubiquitination-mediated regulation of interferon responses. Growth Factors 30, 141-148 (2012).

15. Wang, W. et al. MicroRNA-497 inhibition of ovarian cancer cell migration and invasion through targeting of SMAD specific E3 ubiquitin protein ligase 1. Biochem. Biophys. Res. Commun. 449, 432-437 (2014).

16. Lee, M. G. et al. RASSF1A directly antagonizes RhoA activity through the assembly of a Smurf1-mediated destruction complex to suppress tumorigenesis. Cancer Res. 76, 1847-1859 (2016).

17. Zhang, Y. et al. TRIM52 up-regulation in hepatocellular carcinoma cells promotes proliferation, migration and invasion through the ubiquitination of PPM1A. J. Exp. Clin. Cancer Res. 37, 116 (2018).

18. Luo, D. W. et al. UPP mediated diabetic retinopathy via ROS/PARP and NFkappaB inflammatory factor pathways. Curr. Mol. Med. 15, 790-799 (2015).

19. Dahiya, N., Sherman-Baust, C. A., Wang, T. L. \& Davidson, B. \& Shih le, M. \& Zhang, $Y$. et al. MicroRNA expression and identification of putative miRNA targets in ovarian cancer. PLOS ONE 3, e2436 (2008).

20. Lu, X., Wang, J., Shan, X. \& Li, Y. Selecting key genes associated with ovarian cancer based on differential expression network. J. BUON. 22, 48-57 (2017)

21. Chen, $Y$. et al. MDM2 promotes epithelial-mesenchymal transition and metastasis of ovarian cancer SKOV3 cells. Br. J. Cancer 117, 1192-1201 (2017).

22. Zhao, R. et al. DDB2 modulates TGF-beta signal transduction in human ovarian cancer cells by downregulating NEDD4L. Nucleic Acids Res. 43, 7838-7849 (2015).

23. Langstraat, C., Aletti, G. D. \& Cliby, W. A. Morbidity, mortality and overall survival in elderly women undergoing primary surgical debulking for ovarian cancer: a delicate balance requiring individualization. Gynecol. Oncol. 123, 187-191 (2011).

24. Yao, F. et al. Recurrent fusion genes in gastric cancer: CLDN18-ARHGAP26 induces loss of epithelial integrity. Cell Rep. 12, 272-285 (2015).

25. Holst, M. R. et al. Clathrin-independent endocytosis suppresses cancer cell blebbing and invasion. Cell Rep. 20, 1893-1905 (2017).

26. Bommareddy, A. et al. Alpha-santalol, a component of sandalwood oil inhibits migration of breast cancer cells by targeting the beta-catenin pathway. Anticancer Res. 38, 4475-4480 (2018).

27. Deng, S., Wong, C. K. C., Lai, H. C. \& Wong, A. S. T. Ginsenoside-Rb1 targets chemotherapy-resistant ovarian cancer stem cells via simultaneous inhibition of Wnt/beta-catenin signaling and epithelial-to-mesenchymal transition. Oncotarget 8, 25897-25914 (2017).

28. Gao, Y. et al. Overexpression of RNF146 in non-small cell lung cancer enhances proliferation and invasion of tumors through the Wnt/beta-catenin signaling pathway. PLoS ONE 9, e85377 (2014).

29. Lustig, B. \& Behrens, J. The Wnt signaling pathway and its role in tumor development. J. Cancer Res. Clin. Oncol. 129, 199-221 (2003).

30. Kim, J. G. et al. Wnt3A induces GSK-3 $\beta$ phosphorylation and $\beta$-catenin accumulation through RhoA/ROCK. J. Cell. Physiol. 232, 1104-1113 (2017).

31. Rodrigues, P. et al. RHOA inactivation enhances Wht signalling and promotes colorectal cancer. Nat. Commun. 5, 5458 (2014). 